\title{
Measuring Trans Fats in Native American Adolescents
}

\author{
Madelyn Bradley ${ }^{1}$, Justin Do ${ }^{2}$, Juleene Moritz ${ }^{1}$ and Shasha Zheng ${ }^{3 *}$ \\ ${ }^{1}$ Department of Nutrition Science, California Baptist University, USA \\ ${ }^{2}$ Department of Biological Sciences, University California San Diego, USA \\ ${ }^{3}$ Department of Health Sciences, California Baptist University, USA
}

*Corresponding Author: Shasha Zheng, Department of Health Sciences, California

Baptist University, USA.
Received: June 19, 2021

Published: July 28, 2021

(C) All rights are reserved by Shasha Zheng., et al.

\begin{abstract}
Obesity is becoming increasingly prevalent among US adolescents due to various factors such as food preference, availability, and social condition: however, rates of obesity in Native American adolescents are comparatively much higher than their counterparts as differences of as much as $7.2 \%$ have been reported between Native American and white adolescents. Given extensive study highlighting the highly harmful effects to overall health that consuming trans fats has been linked to, this study investigated the dietary intake levels of trans fats by Native American adolescents and explored the implications in regard to their health in terms of BMI. This was done by collecting macronutrient intake levels of adolescents aged 14-18 at Sherman Indian High School. This information was collected from participants using the Harvard School of Public Health Adolescent Questionnaire (HSPH YAQ) for an eight-week period, the data then sorted participants into appropriate dietary groups using statistical software from Cal Baptist University (CBU). The results of the analysis indicated that there was a statistically significant difference in mean trans fats intake between no risk groups (normal and underweight) and at-risk groups (overweight and obese) ( $\mathrm{s}=0.05$ ) in male adolescents, however statistically significant findings for Native American female adolescents were not observed. This suggests that trans fats intake could be contributing to the high levels of obesity among Native American adolescents. Furthermore, it is of note that this study shows that production and consumption of trans fats are still happening on a day-to-day basis, and a continued effort must be made to eliminate all foods with trans fats from being manufactured and distributed.
\end{abstract}

Keywords: Native American; Adolescents; Obesity; Nutrition; Trans Fats; Dietary Intake

\section{Introduction}

The rate of obesity has been increasing in recent years, especially in the adolescent population. According to the Center for Disease Control and Prevention, the prevalence of obesity was $18.5 \%$ in US youth during the 2015-16 study period [1], increasing from $17.0 \%$ from 2010-2014 [2]. The research also indicated an increase with age, with prevalence rising from $13.9 \%$ among 2 - to 5-year-olds to $20.6 \%$ among 12 - to 19 -year-olds. Although the increase can be seen in various ethnicities, a disparity was found between the US and American Indian or Native American adolescents, where the latter had higher obesity rates than the former [3]. According to a High School Youth Behavior Risk study conducted by the Center for
Disease Control and Prevention (CDC), the rates of obesity among the white students were $13.1 \%$ and $21.3 \%$ for Native Americans [4], a difference that is concerning and worth investigating. In our previous study utilizing the same survey data used in this study, it was discovered that the obesity rates at the Native American school surveyed had doubled. Furthermore, findings from the Maternal and Child Health Journal stated that Native American children are the most obese racial/ethnic group in the U.S. [5]. Oddly, studies among this population group are few, as far more research tends to study the correlation between adult obesity and trans-fats rather than adolescents [6-8]. There have been manuscripts written analyzing the reasons behind why there is a disproportionate level of 
disease in this ethnic group, suggesting that history, culture, and traditional diets might contribute to high obesity levels [9], and in Brazil a study was conducted among overweight and obese adolescents investing the correlation between high saturated fats intake and dyslipidemia [10]. However, there is a lack of research conducted on macro and micronutrient intake among Native American adolescents. In our previous studies, we analyzed the implications of the dietary intake of micronutrients such as calcium, phosphorus, magnesium, sodium, zinc, selenium [11-13]. Research into the macronutrient levels had yet to be conducted, therefore this study found it relevant to investigate the trans fats intake levels among this population.

Trans fatty acids (TFAs) are unsaturated and contain at least one trans double bond, resulting in a straighter formation than the healthier cis-bond fatty acids [14]. Trans fats can be found in animal products, but are most often the products of hydrogenation, a process used to increase fats and oils' shelf-life. This process changes the chemical bond of the fatty acids in the fat from a cis bond into a trans bond, and the most common form of TFAs found in food products are trans-octadecenoic acid isomers, which can be industrially created or naturally created [15]. This process of hydrogenation helps prevent rancidity and provide texture and flavor that is often found desirable in fried or packaged foods [16]. Hydrogenation is cost-effective and convenient for commercial use, especially for frying. Initially, trans fats were actually thought of as a healthy alternative to animal fats due to their unsaturated nature. Scientists disproved this theory in 1990 however, as they discovered that intake of trans fats was linked to an increase in LDL (bad) cholesterol [17]. Trans fats and hydrogenated oils are present in a variety of foods including movie-popcorn, potato and corn chips, sausage rolls, french fries, wedges, battered fish, nuggets, spring rolls, crumbed chicken, fried pastries, pizzas, and packaged desserts to name a few [16]. The health effects of trans fats in the body are alarming. Several studies have indicated that trans-fats increase systemic inflammation, the risk of diabetes, insulin resistance, and cognitive decline [14]. Carvalho., et al. [18] demonstrated that trans fats could cause dyslipidemia. One study [8] even revealed that trans fats intake during pregnancy could cause the body to replicate anxiety-like responses, altering proinflammatory cytokines in the hippocampus of the offspring. A manuscript [19] recently published advocated for the ban of all industrially created trans fats by 2023, arguing that their removal could prevent up to 17 million deaths by 2040 . Unfortunately, the consumption of trans fats is still happening regularly, resulting in poor health and higher risk of disease.
The CDC has a specific goal in Health People 2030 to "Eliminate health disparities, achieve health equity, and attain health literacy to improve health and well-being of all" [20]. Given this goal and the lack of research conducted around the Native American adolescent population, the purpose of this study was to investigate the intake of trans fats among Native American adolescents to help provide potential answers for this ethnic group's high obesity rate.

\section{Research Methods}

\section{Sample subjects}

The data referenced in this study was taken from Sherman Indian High School, a Native American boarding school located in Riverside, California. It opened as early as 1892 under the name of the Perris Indian School, before it was relocated to Riverside in 1903. This study was conducted by Harvard University and several other publications have utilized this information [11-13]. This study used a random sampling method that drew from the population offered by the school; students in grades 9 through 12, representing 43 tribes from across the U.S. The adolescent subjects selected were between the ages of 14-18, and a total of 183 students were interviewed. No other participants of varying ethnicities or schools were utilized, limiting potential bias. The researchers received ethical permission to conduct the study, and all data collectors participated in a mandatory orientation session educating them on the cultural aspects of the population, including studying the best ways to collect the data from the subjects [11].

The data collected and used in this study is anthropometric in nature to examine the prevalence of obesity and analyze how intake levels of trans fats correlates with their health. The anthropometric data taken to determine the subject's health included height $(\mathrm{m})$, weight $(\mathrm{kg})$, waist circumference $(\mathrm{cm})$, and skinfold measurements ( $\mathrm{mm}$ ) which included the calf and triceps. Also calculated were body mass index (BMI) values $\left(\mathrm{kg} / \mathrm{m}^{2}\right)$ and compared to values from the Third National Health and Nutrition Examination Survey (NHNESIII). To obtain the dietary intake data, a semiquantitative food frequency method was used in the format of the Harvard School of Public Health Adolescent Questionnaire (HSPH YAQ). This questionnaire is 12 pages in length with 197 questions and was created with a specific concentration on foods eaten by this age group. There were preliminary focus groups whose goals were to confirm that the foods used in the questionnaire were culturally appropriate. Trained teams collected data over an 8-week period in each of the four dormitories, measuring both macro and micronutrients [11]. 


\section{Statistical analysis}

The data for this study was first converted through Harvard professional software without artificial bias and was then analyzed by California Baptist University using statistical software to calculate the mean daily intakes of trans fats. The t-test was deemed appropriate for this study as t-tests allow for comparing group's data sets to find differences that are statistically significant. One-tailed ttests were used due to the data being continuous and numerical as well as allowing each group mean to be compared to a single other known mean, both means coming from a specific population. This test was utilized to find statistical significance between means expressed as mean \pm SEM. The significance testing was set at $\mathrm{p}$-value $<0.05$. The means were compared between various weight groups according to BMI (underweight, normal, overweight, obese), as well as between male and female populations and to the recommended dietary allowance (RDA).

\section{Results and Discussion}

Males

This study analyzed the intake of total fats in Native American adolescents age 14-18 as well as multiple categories of fats including holistic fat intake, trans fats, saturated fats, trans-polyunsaturated fats, polyunsaturated fats, trans-monounsaturated fats, monounsaturated fats, and fats in relation to daily calorie intake. Of the classes of fats examined, only results from trans-fats proved to be statistically significant. The data from each sex was obtained and the differences between each of the weight categories identified and recorded as a $\mathrm{p}$ value, $\leq .05$ showing significance. Among male subjects age 14 - 18, tests showed that trans-fat intake levels were more elevated in the at-risk weight classes (overweight, obese). Figure 1 displays the trend of greater mean intakes of trans fats in male adolescents in the higher weight classes, with the overweight category consuming the greatest amount. The tests showed that there was great statistical significance $(p \leq 0.05)$ found when comparing a higher weight group to a lower one. Tests showed that the difference between underweight (Mean $=0.54, \mathrm{SEM}=0.28$, df $=3$, $\mathrm{t}$-value $=2.35)$ and obese subjects $($ Mean $=1.67, \mathrm{SEM}=0.36$, $\mathrm{df}=16, \mathrm{t}$-value $=1.74)$ was statistically significant $(\mathrm{p}=0.01)$, and underweight and overweight categories (Mean $=2.62$, SEM $=1.16, \mathrm{df}=6, \mathrm{t}$-value $=1.94)$ reflected similar results $(\mathrm{p}=0.03)$. Even between the lower two weight categories, underweight and normal subjects (Mean $=1.36, \mathrm{SEM}=0.45, \mathrm{df}=18$, t-value $=1.73$ ), the difference between these two groups was statistically signifi- cant $(p=0.05)$. The groups that were not statistically significant when compared were overweight to obese $(p=.08)$, underweight to overweight $(p=.07)$, and normal to obese $(p=.13)$. These results indicate that between each of these different dyads of weight classes, trans-fat intake makes a substantial difference. Notably, the difference between two adjacent weight classes such as Normal and Underweight is still statistically significant, indicating that increased trans-fat levels may be descriptive of the causal relationship between progression in weight classes and the onset of clinical obesity in male adolescents.

\section{Females}

Among females, though the mean intake of trans fat per gram was higher in the overweight and obese categories as is displayed in figure 2 , the tests found there to be no significance between intake levels in weight category comparison. As there was only 1 subject in the underweight $($ Mean $=0.6, \mathrm{SEM}=\mathrm{N} / \mathrm{A}, \mathrm{df}=0, \mathrm{t}$-value $=$ $\mathrm{N} / \mathrm{A}$ ) category for females, a $\mathrm{T}$ test was not applicable for comparisons to this category. For the other three comparisons, the results are as follows: normal (Mean $=0.74, \mathrm{SEM}=0.36, \mathrm{df}=19$, $\mathrm{t}$-value $=$ 1.72 ) to overweight $($ Mean $=0.1, \mathrm{SEM}=.35, \mathrm{df}=13$, $\mathrm{t}$-value $=1.77$ ) $(\mathrm{p}=0.16)$, over to obese (Mean $=0.84, \mathrm{SEM}=0.27, \mathrm{df}=22$, $\mathrm{t}$-value $=1.71)(p=0.23)$, normal to obese $(p=0.31)$. While it is clear there is a higher mean intake of trans fats in higher weight classes in females, the mean difference in the amounts is not great enough to be of significance.

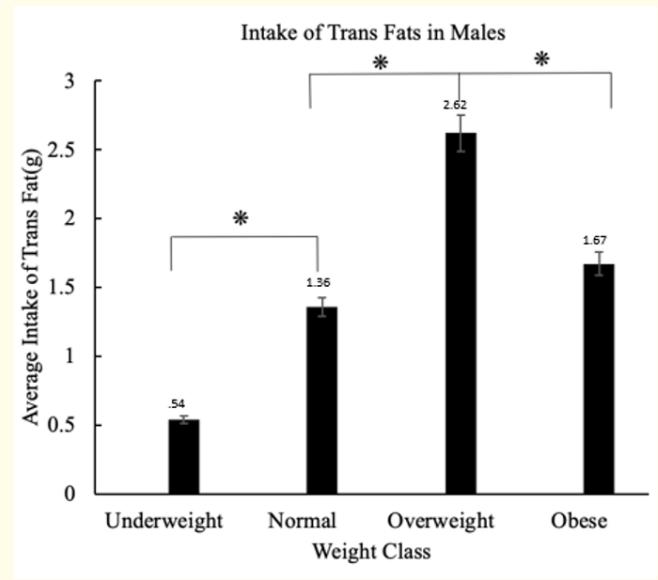

Figure 1: Dietary intake of trans fat across weight groups among male Native American adolescents. Bar indicates mean intake in each weight class \pm SEM. $*$ indicates a significant $\mathrm{p}$-value $<0.05$. 


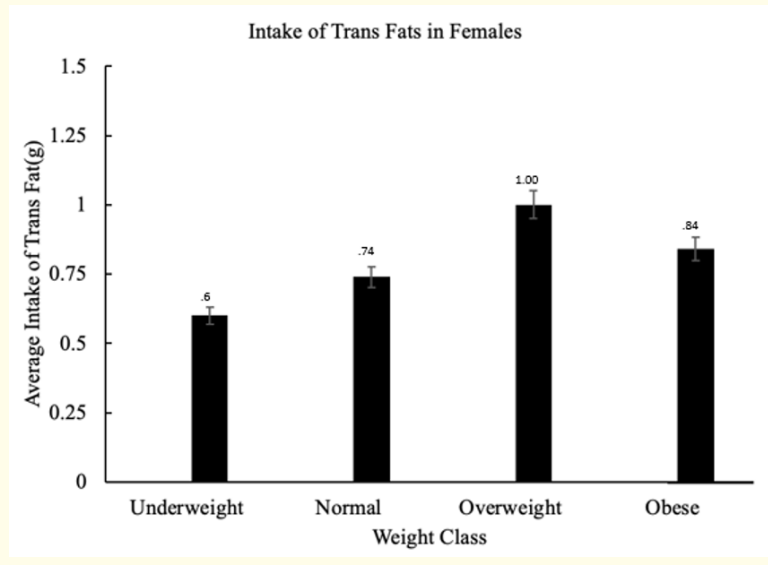

Figure 2: Dietary intake of trans fat across weight groups among female Native American adolescents. Bar indicates mean intake in each weight class \pm SEM.

\section{Discussion}

The results from this study clearly show that there is a statistically significant relationship of trans fats intake and a higher weight category among Native American male adolescents. It is of some note that trans fats intake doesn't have as significant an effect on female weight gain as it does males. A future study could be done to examine the biological mechanisms that lead to this ostensible trend. However, if the mean intake between each weight category is compared male to female, the intake is greater among males in every category, except in the underweight category (Figure 1 and 2). The American Heart Association recommends that less than $1 \%$ of your total daily calories should come from trans fats, and ideally wouldn't be consumed at all [21]. Of the population group in this study, only 14 out of 105 subjects didn't consume trans fats at all. Only one of those 14 being male. This shows a trend towards higher intake of trans fats among males and could contribute to the reason why only the male category showed a positive correlation between grams of trans fat consumed and BMI. A future study that would be helpful would be to collect randomized data on trans fats intake levels across men and women from all ethnic groups in the U.S. Would the results reflect the results from this study, with men having a higher trans fats intake level? A study conducted in 2016 by Harvard University and Tufts University [22] associates attempted to analyze trans fats intake and effects worldwide, but noted it was difficult to do based on the lack of data among coun- tries, sex, and age groups. It is clear that this in an area where data is inadequate, but vitally necessary. It would also be conducive to recreate this study at a second Native American boarding school to see if the trans fats intake trends are analogous or not.

Continuing in this line of thought is the data that shows significantly higher rates of heart disease in men than women. Is it due to reasons such as a larger intake of trans fats as this study notes, and or other nutrients that are harmful for heart health? Or are there other physiological differences that make males more susceptible to heart disease than females? A recent study [23] on sexual dimorphism analyzed the epigenetic mechanisms that the intake of trans fats had in males versus females. It showed that trans fats induced more than twice as many cardiac differentially expressed genes (DEGs) in males in comparison to females. It also downregulated four times as many genes, many of which were connected to specific pathways that lead to hypertrophy, such as Gata4 and Mef2d. This shows that trans fats have detrimental impacts to a person's health from weight gain to heart disease, specifically in males. However, one study [24] conducted with the purpose to examine the correlation between trans fats intake and opioid addiction showed that early trans fats intake consumed in early life stages may lead to a greater chance of opioid addiction, with females having a greater disposition to this. It therefore appears that trans fats do affect each gender uniquely, though it is clearly harmful for both.

Awareness of the harmful effects of trans fats among consumers in the United States has grown in recent years. One study tracked growth in consumer knowledge from 2006 to 2007. Over that oneyear period the percentage of consumers whose awareness grew unaided went from $31 \%$ to $38 \%$ [25]. A study done by the American Heart Association (AHA) [21] tracked trans-fat intake from 1980 2009 and saw the American's consumption of trans fats dropped by one third. PsycTESTS [26] have even created a questionnaire specifically for analysis of trans fats knowledge with 26 items assessing a person's understanding, dietary attitude, sociodemographic characteristics, and frequency of consumption. Bleich S., et al. [27] examined the nutrient trends in restaurants from 2013 - 2018 and noted that items on the menus have declined steadily in caloric levels over the years, specifically declining in the amount of trans fats and saturated fats per item. On May $14^{\text {th }}, 2018$, the World Health Organization (WHO) called for the ban of all industrially created trans fats by 2023, and the EU and U.S. rallied to this call. The U.S. had already contributed to this cause by instigating a mandatory 
labeling of all items containing trans fats in 2003 [28]. However, according to the WHO, only 14 countries have complete or partial bans on trans fats [29]. There are many professionals that are calling for there to be a specific time limit for the food industry in regard to full elimination of trans fats [30]. It is also clear from studies like this one that consumption of trans fats is still happening daily even in the U.S.

A limitation of this study is that the data collected was singularly quantitative. It would be of value to conduct a study with this same population group dedicated to analyzing the subject's knowledge on the negative effects of consuming trans fats. Are the subjects educated on the harmful effects of trans fats and continue to consume them nevertheless, or is there a lack of knowledge on this subject?

\section{Conclusion}

This study set out to examine the trans fats intake levels among the high-risk, little studied, population group of Native American adolescents. The research showed that the daily intake of trans fats is occurring in the Native American adolescent population and could be having harmful effects in terms of BMI and increased health risks, particularly in males. Continued education should be given in schools on the harmful effects trans fats can have, and future studies into the demographics most likely to consume it should be conducted. Understanding the role of trans fat intake in catalyzing the onset of obesity can be crucial in nutrition studies and can be used as precautionary indicators of declining health. Furthermore, continued efforts should be put forth to completely eliminate trans fats in all manufactured foods.

\section{Acknowledgements}

We would like to thank Dr. Margaret Barth for her help on the raw data collection.

\section{Bibliography}

1. "Child obesity facts". Center for Disease Control and Prevention, 24-June-2019 (2020).

2. CL Ogden., et al. "Prevalence of Obesity Among Youths by Household Income and Education Level of Head of Household - United States 2011-2014". MMWR. Morbidity and Mortality Weekly Report (2018).

3. A Bullock., et al. "Obesity and overweight in American Indian and Alaska native children, 2006-2015". American Journal of Public Health 107.9 (2017).
4. "CDC releases 2019 Youth Risk Behavior Survey Results". Centers for Disease Control and Prevention (2020).

5. M Ness., et al. "Correlates of overweight and obesity among American Indian/Alaska native and non Hispanic white children and adolescents: National survey of children's health, 2007". Maternal and Child Health Journal 16.2 (2012): 268277.

6. J Xu., et al. "Macronutrient intake and glycemic control in a population-based sample of American Indians with diabetes: the Strong Heart Study". The American Journal of Clinical Nutrition 86.2 (2007): 480-487.

7. J Aguayo-Aermendariz., et al. "Central obesity and body fat, but not body mass index, are associated with the Pro12Ala polymorphism in the perozisome proliferator-activated receptor $y$ gene in a population with a high consumption of saturated and trans-fatty acids". Nutrition Research (2018): 28-35.

8. C Pase., et al. "Maternal trans fat intake during pregnancy or lactation impairs memory and alters BDNF and TrkB levels in the hippocampus of adult offspring exposed to chronic mild stress". Physiology and Behavior 169 (2017): 114-123.

9. M Fialkowski., et al. "The relevancy of community-based methods: using diet within Native American and Alaska Native adult populations as an example". Clinical and Translational Science 5 (2012): 295-300.

10. L Carvalho., et al. "Consumption of trans fats and saturated fats associated with dyslipidemia in obese and overweight adolescents". Revista Chilena de Nutricion 47 (2020): 73-79.

11. H Dai., et al. "Dietary mineral measurements in normal versus obese Native American adolescents". Journal of Racial and Ethnic Health Disparities 7 (2020): 769-775.

12. M Perez., et al. "Obesity prevalence and dietary intake of antioxidants in Native American adolescents". International Journal of Public Health Sciences 5.3 (2016): 222-227.

13. H Dai., et al. "Dietary nutrient intake and obesity prevalence among Native American adolescents". International Journal of Public Health Sciences 7 (2018): 114-199.

14. S Bhardwaj., et al. "Overview of trans fatty acids: Biochemistry and health effects". Diabetes and Metabolic Syndrome Clinical Research and Reviews 5 (2011): 161-164.

15. R Kodali. "Trans fats: replacement solutions". AOCS PresS (2014). 
16. S Pattanayak. "Trans-fats of processed and fried foods - a choice for taste or serious health problems?". Exploratory Animal and Medical Research 9 (2019): 5-14.

17. M Kovac and R Spruk. "Does the ban on trans-fats improve public health? In search of the optimal response policy". Journal of Regulatory Economics 55 (2019): 258-281.

18. C Carvalho., et al. "Impact of trans-fats on heat-shock protein expression and the gut microbiota profile of mice". Journal of Food Science 83 (2018): 489-498.

19. S Bosch., et al. "Trans fat free by 2023-A building block of the COVID-19 response". Frontiers in Nutrition 8 (2021).

20. Center for Disease Control and Prevention (2021, August 18). Health People 2030.

21. K Doyle. "Americans eating less trans-fat, but still too much". Healthcare and Pharma (2014).

22. Q Wang., et al. "Impact of nonoptimal intakes of saturated, polyunsaturated, and trans fat on global burdens of coronary heart disease". Journal of the American Heart Association 5.1 (2016).

23. K Collison., et al. "Sex-dimorphism in cardiac nutrigenomics: effect of trans fat and/or monosodium glutamate consumption". BMC Genomics 12.555 (2011).

24. K Roversi., et al. "Trans fat intake across gestation and lactation increases morphine preference in females but not in male rats: Behavioral and biochemical parameters". European Journal of Pharmacology 788 (2016): 210-217.

25. R Eckel., et al. "American's awareness, knowledge, and behaviors regarding fats: 2006-2007". Journal of the American Dietetic Association 109.2 (2009): 288-296.

26. S Jasti and S Kovacs. "Trans fat knowledge and food label use measure". PyschTests (2010).

27. S Bleich., et al. "Calorie and nutrient trends in large US chain restaurants, 2012-2018". PLOS ONE 15.2 (2020).

28. S Bloks. "The regulation of trans fats in food products in the US and the EU". Utrecht Law 15.3 (2019).

29. "Public health round-up". Bulletin of the World Health Organization 98.10 (2020): 649.

30. I Pravst. "Speed up global ban on industrial trans fats in food". Nature 560.7718 (2018): 307.
Volume 5 Issue 8 August 2021

(C) All rights are reserved by Shasha Zheng., et al. 